\title{
Spotlight on the 9-valent HPV vaccine
}

This article was published in the following Dove Press journal:

Drug Design, Development and Therapy

20 December 2016

Number of times this article has been viewed

\section{Pier Luigi Lopalco}

Department of Translational Research and New Technologies in Medicine and Surgery, University of Pisa, Pisa, Italy
Correspondence: Pier Luigi Lopalco Department of Translational Research and New Technologies in Medicine and Surgery, University of Pisa, Via San Zeno 35, 56123 Pisa, Italy

Tel +380502213586

Email pierluigi.lopalco@unipi.it
Abstract: Starting in 2006, vaccination against human papillomavirus (HPV) has been progressively implemented in most developed countries. Two vaccines have been successfully used, a bivalent vaccine targeting HPV-related cancers (bHPV) and a quadrivalent vaccine (qHPV) targeting both HPV-related cancers and genital warts. Between December 2014 and June 2015, a new nonavalent HPV vaccine (9vHPV) was granted marketing authorization in the USA and Europe. The 9vHPV was developed from the qHPV and includes five additional HPV types that should increase the level of protection toward HPV-related cancers. Efficacy and/or immunogenicity of 9vHPV has been assessed in eight clinical studies. The 9vHPV vaccine induced a very robust immune response against all vaccine types, with seroconversion rates close to $100 \%$. The safety profile of 9vHPV is comparable to that of qHPV. Local reactions, especially swelling, have been more frequently reported after 9vHPV than qHPV, and this slightly increases when the $9 \mathrm{vHPV}$ is coadministered with other vaccines. The additional coverage offered by the $9 \mathrm{vHPV}$ may prevent a significant proportion of HPV-related cancers (variable between $8 \%$ and 18\%) depending on the local distribution of high-risk HPV types in the population. It is impossible, at present, to anticipate the actual impact of the wide use of the 9vHPV in comparison with the bHPV or the qHPV, since it depends on many variables including duration of protection, potential cross-protection toward nonvaccine types, and herd immunity effect.

Keywords: human papillomavirus vaccine, immunogenicity, vaccine safety, cervical cancer, head and neck cancer, genital warts

\section{Background}

In 1842, the Italian physician Rigoni-Stern, ${ }^{1}$ in Verona, observed that deaths after cervical cancer seldom occurred in virgins and nuns, being more frequent in married women, widows, and prostitutes. This was the first observation suggesting a link between sexual contact and cervical cancer. But it was only in the early 1970s that scientific experiments tried to establish a causal relationship between human papillomavirus (HPV) infection and cervical cancer. zur Hausen ${ }^{2,3}$ received the Nobel Prize in Medicine in 2008 for his studies on the oncogenic role of HPVs. Based on epidemiological observation, Muñoz et $\mathrm{al}^{4}$ in 2003 listed 15 HPV types $(16,18,31$, $33,35,39,45,51,52,56,58,59,68,73$, and 82) as high-risk types with a clear carcinogenic role, forming the basis for an epidemiological classification of HPV types associated with cancer. To date, the World Health Organization's International Agency for Research on Cancer (IARC) has classified twelve HPV types (16, 18, 31, 33, 35, $39,45,51,52,56,58$, and 59) among the "Group 1 carcinogens". 5

As a result of the continuous progress in this field, two vaccines against HPV infection have been developed. A quadrivalent vaccine (qHPV) gained approval from the US Food and Drug Administration (FDA) in 2006 and also received a positive opinion from the European Medicine Agency. A bivalent vaccine (bHPV) received a positive opinion from the European Medicine Agency in 2007 and was approved 
Table I Summary characteristics of the licensed vaccines against HPV infection

\begin{tabular}{|c|c|c|}
\hline Vaccine - product name & HPV types & Vaccine composition \\
\hline \multirow{5}{*}{ bHPV - Cervarix ${ }^{\circledR}$} & $16-18$ & Type I6 LI protein $20 \mu \mathrm{g}$ \\
\hline & & Type I8 LI protein $20 \mu \mathrm{g}$ \\
\hline & & Adjuvanted by AS04, containing: \\
\hline & & $-M P L^{a} 50 \mu g$ \\
\hline & & - Adsorbed on $\mathrm{Al}(\mathrm{OH})_{3} 0.5 \mathrm{mg}$ \\
\hline \multirow[t]{6}{*}{ qHPV - Gardasil ${ }^{\circledast} /$ Silgard $^{\circledR}$} & $6-11-16-18$ & Type $6 \mathrm{LI}$ protein $20 \mu \mathrm{g}$ \\
\hline & & Type II LI protein $40 \mu \mathrm{g}$ \\
\hline & & Type $16 \mathrm{LI}$ protein $40 \mu \mathrm{g}$ \\
\hline & & Type $18 \mathrm{LI}$ protein $20 \mu \mathrm{g}$ \\
\hline & & Adsorbed on amorphous aluminum \\
\hline & & hydroxyphosphate sulfate adjuvant ( $225 \mu \mathrm{g})$ \\
\hline \multirow[t]{11}{*}{ 9vHPV - Gardasil-9 ${ }^{\circledR}$} & $6-||-|6-| 8-3 \mid-33-45-52-58$ & Type $6 \mathrm{LI}$ protein $30 \mu \mathrm{g}$ \\
\hline & & Type II LI protein $40 \mu \mathrm{g}$ \\
\hline & & Type $16 \mathrm{LI}$ protein $60 \mu \mathrm{g}$ \\
\hline & & Type I $8 \mathrm{LI}$ protein $40 \mu \mathrm{g}$ \\
\hline & & Type 3I LI protein $20 \mu \mathrm{g}$ \\
\hline & & Type $33 \mathrm{LI}$ protein $20 \mu \mathrm{g}$ \\
\hline & & Type $45 \mathrm{LI}$ protein $20 \mu \mathrm{g}$ \\
\hline & & Type $52 \mathrm{LI}$ protein $20 \mu \mathrm{g}$ \\
\hline & & Type $58 \mathrm{LI}$ protein $20 \mu \mathrm{g}$ \\
\hline & & Adsorbed on amorphous aluminum \\
\hline & & hydroxyphosphate sulfate adjuvant ( $500 \mu \mathrm{g})$ \\
\hline
\end{tabular}

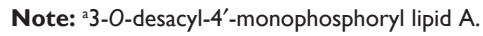

Abbreviation: HPV, human papillomavirus.

by the FDA in 2009. Both qHPV and bHPV include HPV types 16 and 18 , which represent the most common cause of cervical cancer. In addition, qHPV includes HPV types 6 and 11, which are responsible for the majority of genital warts. The characteristics of the two vaccines are summarized in Table 1.

To date, most of the industrialized countries have introduced HPV vaccination. ${ }^{6} 7$ In addition, GAVI started the first HPV vaccine demonstration program in Kenya in 2013, thus vaccinating 300,000 girls and helping seven countries initiate other demonstration programs. ${ }^{8}$ Implementation of HPV vaccination programs varies a lot across the countries. Most of the industrialized countries recommend HPV vaccination for at least one cohort of adolescent or preadolescent girls (starting from 9 years of age). Catch-up programs, extended to older girls and women, have been implemented in nine European countries, ${ }^{9}$ USA, ${ }^{10}$ and Canada. ${ }^{11}$ More recently, few countries, including the USA, extended the HPV vaccine recommendation to the male population as well.

In December 2014, a nonavalent vaccine (9vHPV), developed starting from the qHPV, was licensed by the FDA. ${ }^{12}$ The 9vHPV gained marketing authorization from the European Commission, valid throughout the European Union, in June $2015 .{ }^{13}$ The 9vHPV contains type 31, 33, 45, 52, and 58 antigens in addition to those included in the qHPV. The content of 9vHPV is shown in Table 1. The additional highrisk types included in the 9vHPV should provide a higher protection against HPV-related cancers, thus improving the overall impact of the vaccination programs. This paper aims at reviewing the available evidence on safety, immunogenicity, and potential impact of the 9vHPV.

\section{Efficacy and immunogenicity of the 9vHPV}

Efficacy and/or immunogenicity of the 9vHPV was assessed in eight clinical studies, which are summarized in Table 2.

Comparison of 9vHPV against placebo was not acceptable since HPV vaccination is recommended and implemented in many countries with either bHPV or qHPV. Therefore, the strategy used in the clinical development of 9vHPV has been based on the following steps:

Step 1: efficacy of 9vHPV against persistent infection and disease related to HPV types 6, 11, 16, or 18 to be inferred from noninferior immunogenicity studies between 9vHPV and qHPV in girls aged 9-15 years and in women and men aged 16-26 years;

Step 2: efficacy against persistent infection and disease related to the new types included in the 9vHPV 
Table 2 Summary of the clinical studies to evaluate immunogenicity, efficacy, and safety of 9vHPV, in relation to the strategy used for the clinical development ${ }^{\mathrm{a}}$

\begin{tabular}{|c|c|c|c|c|}
\hline $\begin{array}{l}\text { Study } \\
\text { code }\end{array}$ & Objectives & $\begin{array}{l}\text { Strategic } \\
\text { objective }\end{array}$ & Population & References \\
\hline 001 & $\begin{array}{l}\text { Noninferior immunogenicity against HPV types } 6,1 \mathrm{I}, 16 \text {, } \\
\text { and } 18 \text { and efficacy against HPV types } 31,33,45,52 \text {, and } \\
58 \text { of } 9 \mathrm{vHPV} \text { compared to } \mathrm{HHPV} \text { in women } 16 \text { through } \\
26 \text { years of age }\end{array}$ & Steps I and 2 & $\begin{array}{l}\mathrm{N}=14,204: 7,099 \text { receiving } \\
9 \mathrm{vHPV} \text { and } 7,105 \text { receiving } \\
\text { qHPV vaccine }\end{array}$ & Joura et $\mathrm{al}^{14}$ \\
\hline 002 & $\begin{array}{l}\text { Immunogenicity of 9vHPV in girls and boys } 9 \text { through } \\
15 \text { years of age and women } 16 \text { through } 26 \text { years of age }\end{array}$ & Step 3 & $\begin{array}{l}\mathrm{N}=3,066: 1,932 \text { girls; } 666 \\
\text { boys; and } 468 \text { women } \\
\text { receiving 9vHPV }\end{array}$ & Van Damme et $\mathrm{al}^{18}$ \\
\hline 003 & $\begin{array}{l}\text { Immunogenicity of 9vHPV in men } 16 \text { through } 26 \text { years of } \\
\text { age and women } 16 \text { through } 26 \text { years of age }\end{array}$ & Step 3 & $\begin{array}{l}\mathrm{N}=2,5 \mathrm{I} \text { : }: \mathrm{I}, \mathrm{I03} \mathrm{HM} ; 3 \mathrm{I} 3 \\
\text { MSM; and I,099 women } \\
\text { receiving 9vHPV }\end{array}$ & Castellsagué et al ${ }^{19}$ \\
\hline 006 & $\begin{array}{l}\text { Administration of } 9 \mathrm{vHPV} \text { to girls and women } 12 \text { through } \\
26 \text { years of age previously vaccinated with } \mathrm{QHPV} \text { vaccine }\end{array}$ & $\begin{array}{l}\text { 9vHPV } \\
\text { revaccination } \\
\text { after qHPV }\end{array}$ & $\begin{array}{l}\mathrm{N}=921: 615 \text { receiving } 9 \mathrm{vHPV} \\
\text { and } 306 \text { receiving placebo }\end{array}$ & Garland et $\mathrm{al}^{20}$ \\
\hline $\begin{array}{l}005 \text { and } \\
007\end{array}$ & $\begin{array}{l}\text { Concomitant administration of 9vHPV with vaccines } \\
\text { recommended routinely in girls and boys II through } \\
\text { I5 years of age }\end{array}$ & $\begin{array}{l}\text { 9vHPV } \\
\text { coadministration }\end{array}$ & $\begin{array}{l}\mathrm{N}=2,295: 1,147 \text { boys and girls } \\
\text { in the concomitant group and } \\
\mathrm{I}, 148 \text { in the nonconcomitant } \\
\text { group }\end{array}$ & $\begin{array}{l}\text { Schilling et al; }{ }^{21} \\
\text { Kosalaraksa et } \mathrm{al}^{22}\end{array}$ \\
\hline 009 & $\begin{array}{l}\text { Immunogenicity of } 9 \mathrm{vHPV} \text { in girls } 9 \text { through } \\
15 \text { years of age }\end{array}$ & Step I & $\begin{array}{l}\mathrm{N}=600: 300 \text { receiving } 9 \mathrm{vHPV} \\
\text { and } 300 \text { receiving } \mathrm{qHPV} \\
\text { vaccine }\end{array}$ & Vesikari et $\mathrm{al}^{15}$ \\
\hline 010 & $\begin{array}{l}\text { Immunogenicity of two doses of } 9 \mathrm{vHPV} \text { in girls and boys } \\
9 \text { through } 14 \text { years of age and three doses of } 9 \mathrm{vHPV} \text { in } \\
\text { girls } 9 \text { through I } 4 \text { years of age and women } 16 \text { through } \\
26 \text { years of age }\end{array}$ & $\begin{array}{l}\text { Two doses } \\
\text { schedule } \\
\text { evaluation }\end{array}$ & $\begin{array}{l}\mathrm{N}=|, 5| 8: 753 \text { girls; } 45 \mid \text { boys; } \\
\text { and } 3 \mid 4 \text { women }\end{array}$ & $\begin{array}{l}\text { European } \\
\text { Medicines Agency }{ }^{17}\end{array}$ \\
\hline 020 & $\begin{array}{l}\text { Evaluated immunogenicity of 9vHPV in men } 16 \text { through } \\
26 \text { years of age }\end{array}$ & Step I & $\begin{array}{l}\mathrm{N}=500: 249 \text { receiving } 9 \mathrm{vHPV} \\
\text { and } 25 \mathrm{I} \text { receiving } \mathrm{qHPV} \\
\text { vaccine }\end{array}$ & Van Damme et al ${ }^{16}$ \\
\hline
\end{tabular}

Notes: aStep I: the efficacy of 9vHPV against persistent infection and disease related to HPV types 6, II, I6, or 18 to be inferred from noninferior immunogenicity studies between 9vHPV and the GHPV vaccine in girls 9-15 years, women and men 16-26 years; Step 2: the efficacy against persistent infection and disease related to the new types included in the 9vHPV $(31,33,45,52$, and 58) to be demonstrated in girls and women 16-26 years; Step 3: the demonstration of noninferior immunogenicity against the 9vHPV types in boys and girls 9-15 years and men 16-26 years, compared to women 16-26 years.

Abbreviations: HPV, human papillomavirus; HM, heterosexual men; MSM, men who have sex with men.

$(31,33,45,52$, and 58$)$ to be demonstrated in girls and women aged $16-26$ years;

Step 3: demonstration of noninferior immunogenicity against the 9vHPV types in boys and girls aged 9-15 years and men aged 16-26 years, compared to women aged 16-26 years (Figure 1).

No direct comparison between 9vHPV and bHPV can be performed in terms of efficacy due to the fact that crossprotection of bHPV toward HPV types not included in the vaccine is widely under debate.

\section{Efficacy of 9vHPV against persistent infection and disease related to types 6 , $\mathrm{II}, 16$, and 18}

Efficacy against types $6,11,16$, and 18 was anticipated to be similar between 9vHPV and qHPV. For this reason,

\section{HPV types \\ $6,11,16$, and 18}

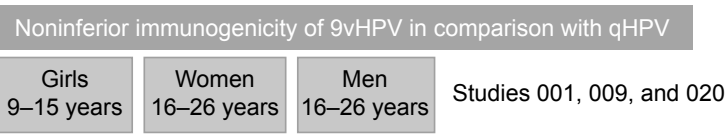

HPV types

$31,33,45,52$, and 58

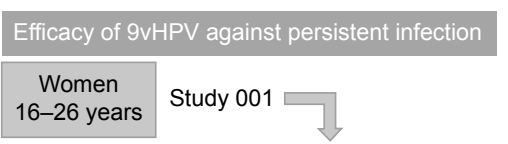

Noninferior immunogenicity in comparison with women $16-26$ years

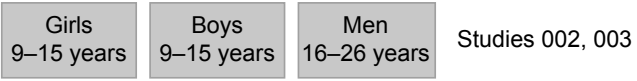

Figure I Strategy followed to assess immunogenicity and efficacy of 9vHPV in premarketing clinical studies.

Abbreviation: HPV, human papillomavirus. 
it was primarily assessed using a bridging strategy that demonstrated noninferior immunogenicity of 9vHPV compared with qHPV in women aged 16-26 years (Study 001), ${ }^{14}$ girls aged 9-15 years (Study 009), ${ }^{15}$ and men aged 16-26 years (Study 020). ${ }^{16}$ Geometric mean titers (GMTs) for anti-HPV 6, 11, 16, and 18 are summarized on a logarithmic scale in Figure 2. No significant difference in GMT has been demonstrated in any age group between 9vHPV and qHPV recipients. Seroconversion rates within 1 month after dose 3 in the 9vHPV populations were between $99.8 \%$ and $100 \%$.

\section{Efficacy of 9vHPV against persistent infection and disease related to types 31 , $33,45,52$, and 58}

Study protocol 001 has been used to assess the efficacy of 9vHPV against persistent infection and disease due to the new types included in the nonavalent vaccine in women aged 16-26 years. ${ }^{14,17}$ A total of 14,204 women (9vHPV =7,099; qHPV $=7,105$ ) were followed up for 67 months after administration of dose 3 , with a median duration of 43 months after dose 3 , in a randomized, double-blind clinical trial.

The per-protocol efficacy (PPE) population included all women who were seronegative on day 1 of the study and had negative results on polymerase chain reaction assays for the relevant HPV type from day 1 through month 7 . High-grade cervical intraepithelial neoplasia, adenocarcinoma in situ, invasive cervical carcinoma, high-grade vulvar intraepithelial neoplasia, high-grade vaginal intraepithelial neoplasia, vulvar cancer, and vaginal cancer were the conditions representing the study end points. The efficacy of $9 \mathrm{vHPV}$ on both persistent infection and disease related to the HPV types $31,33,45,52$, or 58 is summarized in Table 3. In the PPE population, the risk reduction for both persistent infection and low-/high-grade disease has been very high, being close to $100 \%$ for most of the considered end points. Only one case of high-grade disease (CIN 2) occurred in the 9vHPV PPE population, and this was related to HPV type 58 infection. Remarkably, this subject also had positive results for HPV type 56 both at baseline and later on until the time of diagnosis, thus suggesting that coinfection by a HPV type not covered by the 9HPV was a plausible cause of vaccine failure. The incidence of both persistent infection and low-/ high-grade disease related to HPV types 6, 11, 16, and 18 was similar in the 9vHPV and qHPV groups, and no significant risk reduction was detected.

The modified intention-to-treat (ITT) population included women who received at least one dose of vaccine and for whom there was at least one measurement of efficacy for the corresponding end point. The ITT population included both naïve and non-naïve women. The 9vHPV vaccine efficacy toward high-grade disease in the subgroup of nonHPV-infected women was 42.5\% (95\% confidence interval: 7.9-65.9). Disease rates in the subgroup of HPV-infected women were similar in both 9vHPV and qHPV groups.

\section{Noninferior immunogenicity against the 9vHPV types in boys and girls aged 9-15 years and men aged 16-26 years, compared to women aged 16-26 years}

The immune response to $9 \mathrm{vHPV}$ with regard to the new HPV types has been assessed in the younger age groups and in men aged 16-26 years in comparison with women

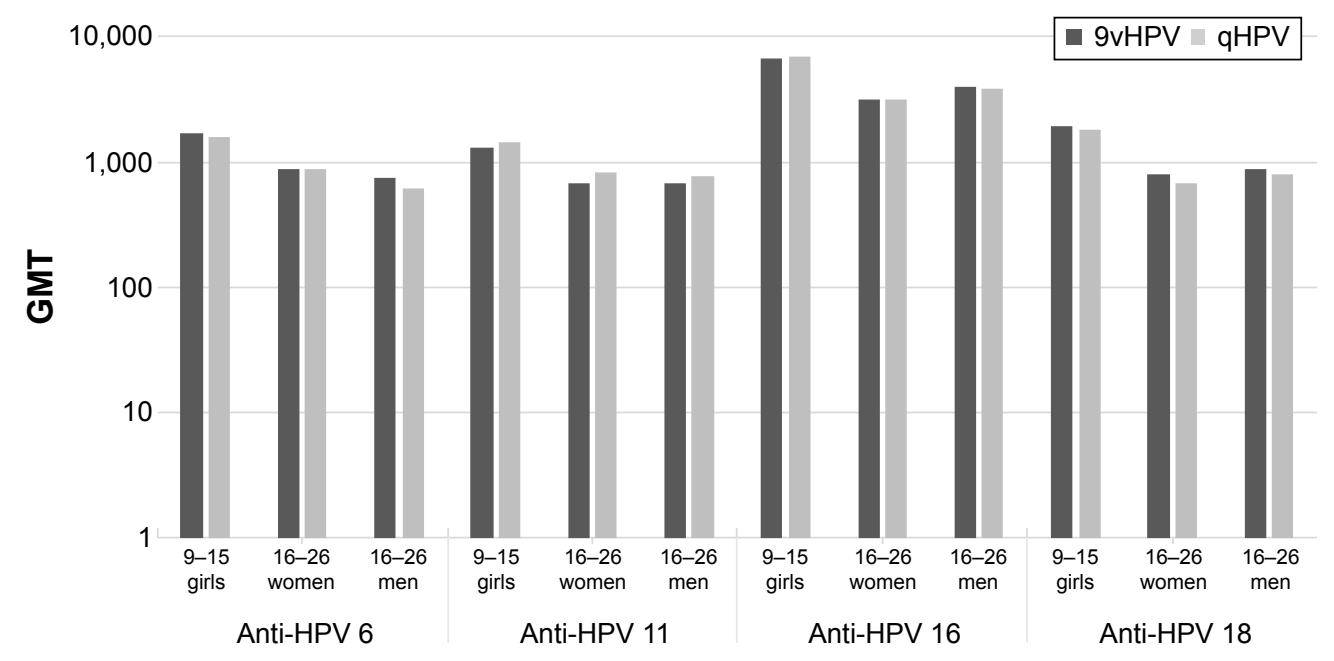

Figure 2 Comparison of immune responses (GMT) between 9vHPV and qHPV vaccine for HPV types 6, 11, 16, and 18 in 9-15 year old girls and I6-26 year old women and men.

Abbreviations: GMT, geometric mean titer; HPV, human papillomavirus. 
Table 3 Efficacy of 9vHPV on persistent HPV infection and on cervical, vulvar, and vaginal disease (HPV types 31, 33, 45, 52, or 58) in the per-protocol efficacy population ${ }^{14}$

\begin{tabular}{|c|c|c|c|c|c|}
\hline \multirow[t]{2}{*}{ Outcome } & \multicolumn{2}{|c|}{ 9vHPV (N=7,099) } & \multicolumn{2}{|c|}{ qHPV $(\mathrm{N}=7,105)$} & \multirow{2}{*}{$\begin{array}{l}\text { Risk reduction } \\
95 \% \mathrm{Cl}\end{array}$} \\
\hline & Cases & $\begin{array}{l}\text { Cases/I,000 } \\
\text { person-years }\end{array}$ & Cases & $\begin{array}{l}\text { Cases/I,000 } \\
\text { person-years }\end{array}$ & \\
\hline \multicolumn{6}{|l|}{ Persistent infection } \\
\hline$\geq 6$ months & 35 & 2.1 & 810 & 52.4 & $96.0(94.4-97.2)$ \\
\hline$\geq 12$ months & 21 & 1.3 & 544 & 34.5 & $96.3(94.4-97.7)$ \\
\hline \multicolumn{6}{|l|}{ Cervical, vulvar, and vaginal } \\
\hline CINI, condyloma, VINI, and VaINI & 2 & 0.1 & 82 & 4.3 & $97.6(91.7-99.6)$ \\
\hline $\mathrm{CIN} 2 / 3, \mathrm{VIN} 2 / 3, \mathrm{VaIN} 2 / 3$, or worse & I & 0.1 & 30 & 1.6 & $96.7(80.9-99.8)$ \\
\hline \multicolumn{6}{|l|}{ Cervical disease } \\
\hline CINI & 1 & 0.1 & 69 & 4.0 & $98.6(92.4-99.9)$ \\
\hline CIN2/3 or worse & I & 0.1 & 27 & 1.5 & $96.3(79.5-99.8)$ \\
\hline \multicolumn{6}{|l|}{ Vulvar and vaginal disease } \\
\hline Condyloma & 0 & 0.0 & 3 & 0.2 & $100(-71.6$ to 100$)$ \\
\hline VINI or VaINI & I & 0.1 & 12 & 0.6 & $91.7(51.3-99.6)$ \\
\hline $\mathrm{VIN} 2 / 3$, ValN2/3, or worse & 0 & 0.0 & 3 & 0.2 & $100(-71.5$ to 100$)$ \\
\hline
\end{tabular}

Note: Adapted with permission from Joura E, Giuliano AR, Iversen O-E, et al. A 9-valent HPV vaccine against infection and intraepithelial neoplasia in women. N Engl J Med. 2015;372(8):71I-723.14

Abbreviation: $\mathrm{Cl}$, confidence interval.

aged $16-26$ years. ${ }^{18,19}$ Seroconversion rates measured at month 7 were close to $100 \%$ in all age groups. ${ }^{17}$ Immunogenicity levels in terms of GMT at month 7 are shown in Figure 3. The immune response to the new antigen types included in the 9vHPV is comparable between the sexes and is slightly higher in the younger age groups in comparison to women and men aged $16-26$ years. On the basis of such bridging immunogenicity evaluation, the efficacy of $9 \mathrm{vHPV}$ against the new vaccine types is inferred from women aged 16-26 years to men aged 16-26 years and younger age groups. ${ }^{17}$

In conclusion, the 9vHPV induced a very robust immune response against all vaccine types, with seroconversion rates close to $100 \%$. GMTs were higher in the younger age groups (boys and girls) than in the men and women aged 16-26 years.

\section{Use of 9vHPV in individuals previously vaccinated with $\mathrm{QHPV}$}

Immunogenicity of 9vHPV in females aged 12-26 years who were previously vaccinated with three doses of qHPV has been assessed in a double-blind, placebo-controlled randomized trial (Study 006). ${ }^{20}$ Between $98.3 \%$ and $100 \%$ of the study population previously vaccinated with qHPV seroconverted to the new types included in the 9vHPV. The level of immune response elicited by 9vHPV in previously

10,000

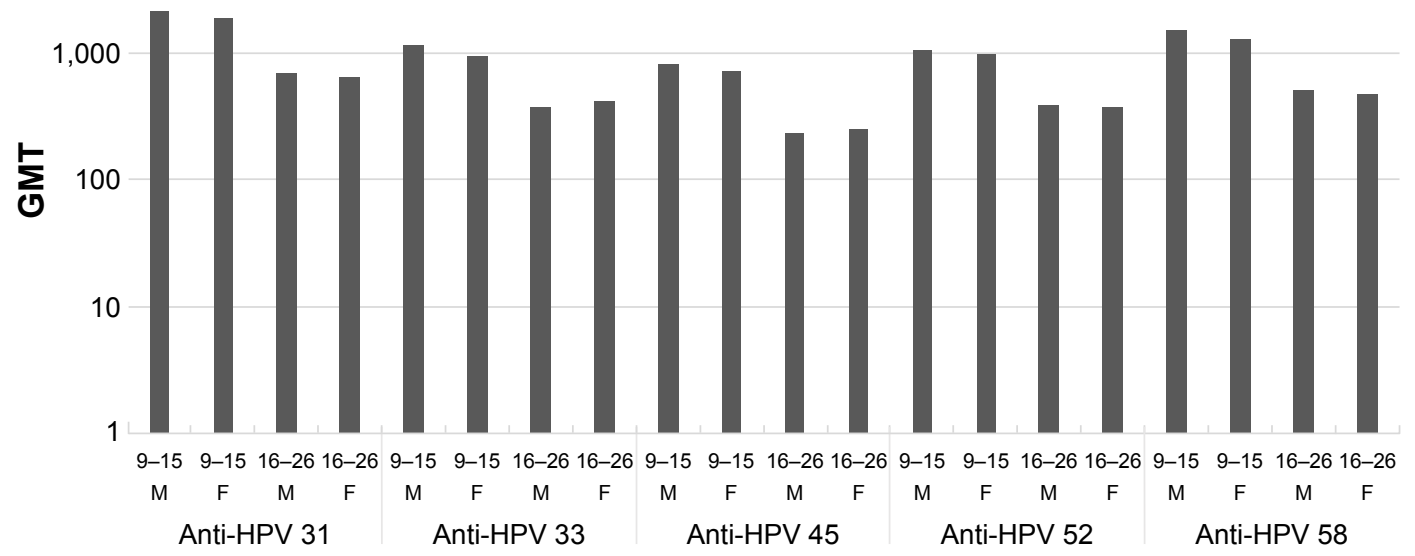

Figure 3 Comparison of immune responses (GMT) at month 7 after 9vHPV vaccination to HPV types 31, 33, 45, 52, and 58 in 9-15 year old girls and boys, and I6-26 year old women and men.

Abbreviations: GMT, geometric mean titer; HPV, human papillomavirus; M, male; F, female. 


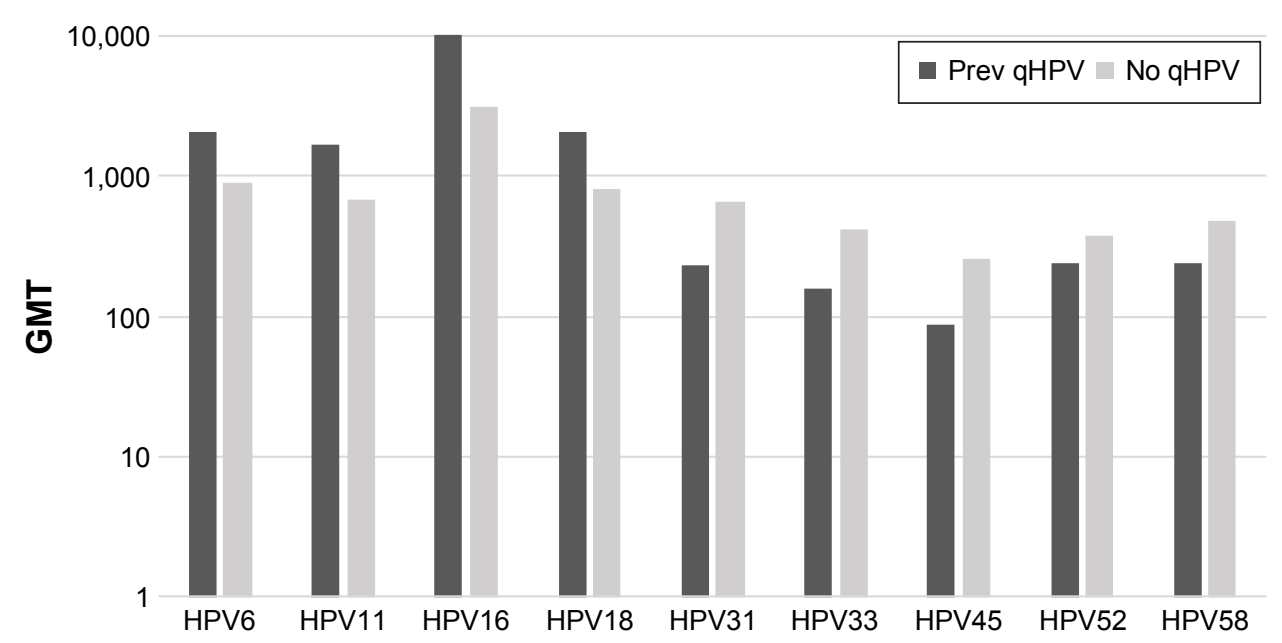

Figure 4 Comparison of immune responses (GMT) at month 7 after 9vHPV vaccination in 16-26 year old women that received or not previous vaccination with qHPV. Abbreviation: GMT, geometric mean titer.

vaccinated individuals was compared to that measured in the pivotal Study 001 in a naïve female population. ${ }^{14}$ The immune response to HPV types 6,11,16, and 18 after vaccination with 9vHPV was higher in women aged 16-26 years who were previously vaccinated with qHPV than in those receiving $9 \mathrm{vHPV}$ only. On the contrary, the immune response to HPV types $31,33,45,52$, and 58 was lower in those previously vaccinated with qHPV (Figure 4). In any case, such an observation is difficult to interpret since there is no established threshold for protection in terms of antibody titers.

\section{Use of 9vHPV with a two-dose schedule}

Immunogenicity of 9vHPV has been assessed in girls and boys aged 9-14 years receiving a two-dose schedule (either 6- or 12-months interval) in comparison to girls aged 9-14 years and women aged 16-26 years receiving three doses at 0,2 , and 6 months. ${ }^{17}$ One month following the last dose of the assigned regimen, seroconversion rates across all groups were between $97.9 \%$ and $100 \%$. GMTs for each of the nine vaccine HPV types were higher in girls and boys who received two doses of $9 \mathrm{vHPV}$ (at either $0-6$ or $0-12$ months) than women aged 16-26 years who received three doses (at 0,2 , and 6 months). On the basis of this immunogenicity bridging, the efficacy of a two-dose regimen of 9vHPV in girls and boys aged 9-14 years was inferred.

In the same study, in girls and boys aged 9-14 years, GMTs at 1 month after the last vaccine dose were numerically lower for some vaccine types after a two-dose schedule than after a three-dose schedule (ie, HPV types 18, 31, 45, and 52 after 0 and 6 months and HPV type 45 after 0 and 12 months). The clinical relevance of these findings is unknown.

\section{Concomitant administration of 9vHPV with other vaccines usually given to adolescents}

The 9vHPV has been coadministered with Neisseria meningitidis serotypes $\mathrm{A} / \mathrm{C} / \mathrm{Y} / \mathrm{W}-135$ or diphtheria/tetanus/ acellular pertussis (Tdap) vaccines in a population of boys and girls aged 9-14 years ${ }^{21}$ and with diphtheria/tetanus/ acellular pertussis/inactivated poliomyelitis vaccine (TdapIPV) in a population of boys and girls aged 11-14 years. ${ }^{22}$ In both studies, the immune response to all component of administered vaccines was noninferior when compared with nonconcomitant administration.

\section{Safety of the 9vHPV}

Safety of the 9vHPV has been assessed during the clinical development in a large population of about 16,000 individuals. ${ }^{17}$ The most common adverse events reported among recipients who received the vaccines were injectionsite adverse reactions and headache, which were mild to moderate in intensity (Table 4). Swelling at the injection site has been more frequently reported among recipients

Table 4 Summary of the safety profile of 9vHPV

\begin{tabular}{ll}
\hline Adverse reaction & Frequency $^{\mathbf{a}}$ \\
\hline Pain, swelling, and redness at the & Very common \\
injection site & \\
Headache & Very common \\
Dizziness & Common \\
Nausea & Common \\
\hline
\end{tabular}

Notes: Adverse reactions reported with a frequency of at least $0.1 \%$ during clinical trials. ${ }^{17}$ aVery common: $\geq 1 / 10$; common: $\geq 1 / 100$ to $<1 / 10$. 


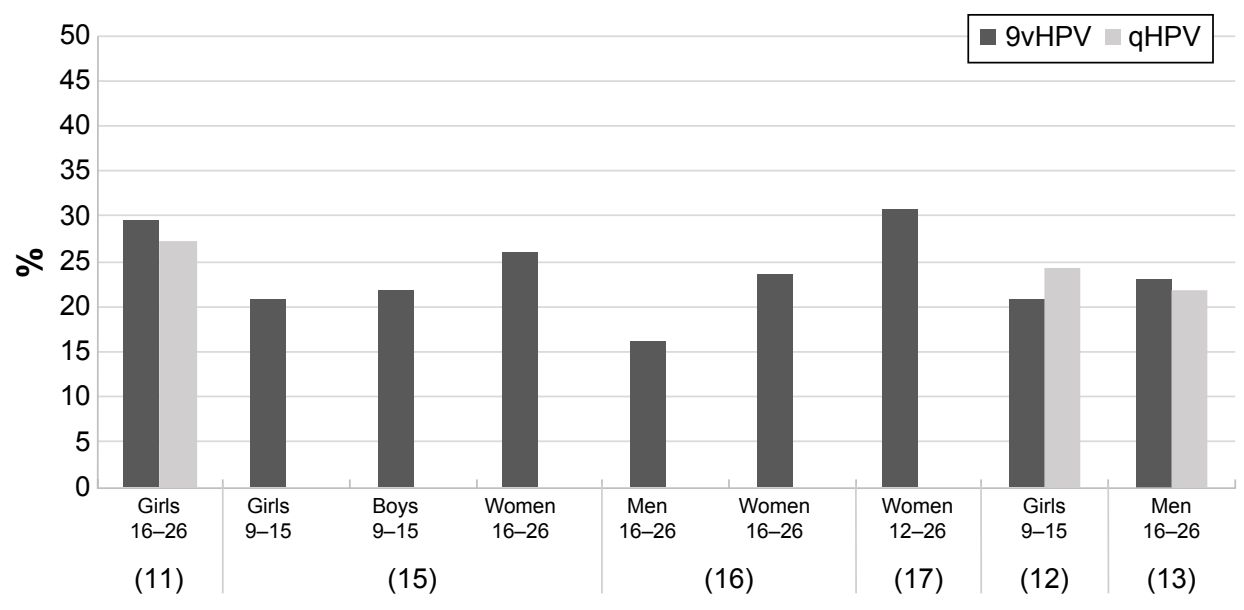

Figure 5 Frequency of vaccine-related systemic adverse events (systemic adverse events included fever, headache, fatigue, nausea, dizziness, oropharyngeal or abdominal pain, myalgia, diarrhea) after 9vHPV and qHPV vaccination (reference citation number).

of the $9 \mathrm{vHPV}$ vaccine than recipients of the qHPV both in women aged $16-26$ years $(40.0 \% \text { vs } 28.8 \%)^{14}$ and girls aged 9-15 years $(47.8 \%$ vs $36.0 \%) .{ }^{15}$

Headache has been the most frequent systemic adverse event related to vaccine administration, followed by pyrexia, nausea, dizziness, and fatigue. The overall frequency of vaccine-related systemic adverse events was similar in 9vHPV and qHPV vaccines (Figure 5).

Three serious adverse events were reported that were considered by the investigators as being related to the vaccination: one case of asthma in a 10-year-old boy, who was hospitalized and then fully recovered; ${ }^{18}$ one case of a 21 -yearold woman who was hospitalized for severe headache and fever, discharged after 2 days with a possible diagnosis of viral infection, and fully recovered after 8 days; ${ }^{18}$ and one case of an 18-year-old woman who experienced purulent tonsillitis 2 days after receiving dose 1 of 9vHPV vaccine, was hospitalized, and then fully recovered. ${ }^{20}$

No report on postmarketing safety data is available to date. In 2014, Centers for Disease Control and Prevention published a report analyzing the events reported to the Vaccine Adverse Event Reporting System (VAERS) following qHPV vaccination from June 2006 through March 2014 . $^{23}$ In that report, about $92 \%$ of the qHPV reports were classified as nonserious. The most commonly reported adverse events were syncope (fainting), dizziness, nausea, headache, fever, and injection-site reactions (pain, swelling, and redness). From a preliminary analysis of the VAERS database, in 2015, excluding reports related to programmatic errors (incorrect product storage, inappropriate schedule, etc), 386 reports of adverse events after 9vHPV have been submitted to VAERS, in comparison to 1,581 reports related to $\mathrm{qHPV}$ (personal observation). The most frequent events reported after 9vHPV vaccination in 2015 were dizziness, syncope, headache, and injection-site reactions.

In conclusion, the safety profile of $9 \mathrm{vHPV}$ is comparable to $\mathrm{qHPV}$, but local reactions, especially swelling, have been more frequently reported after 9vHPV than after qHPV administration. Concomitant administration with other vaccines has been shown to slightly increase the local reactogenicity. Headache and fever have been the most frequent systemic adverse events reported after 9vHPV, with a frequency comparable to qHPV vaccination. ${ }^{17}$

\section{Anticipated impact of the 9vHPV}

The anticipated impact of 9vHPV in comparison with bHPV and qHPV is related to its larger coverage of high-risk HPV types. Since cross-protection against HPV types not included in the vaccine has not been demonstrated, the potential impact of 9vHPV use is strictly associated with the prevalence of disease related to the additional five types covered by the new vaccine. Worldwide, the distribution of HPV types among HPV-related cancers significantly varies. A large review done by Zhai and Tumban ${ }^{24}$ showed that protection given by 9vHPV would cover HPV types associated with $92 \%$ of cervical cancers in North America, 90.9\% in Europe, 86.5\% in Australia, and $89.9 \%$ worldwide. Therefore, the additional coverage provided by the new vaccine against cervical disease would be around 13\% in North America and Europe, 8.8\% in Australia, and 18.4\% worldwide (Figure 6).

The Centers for Disease Control and Prevention's analysis on high-quality data from the National Program of Cancer Registries and the National Cancer Institute's Surveillance, Epidemiology, and End Results program, over the period 


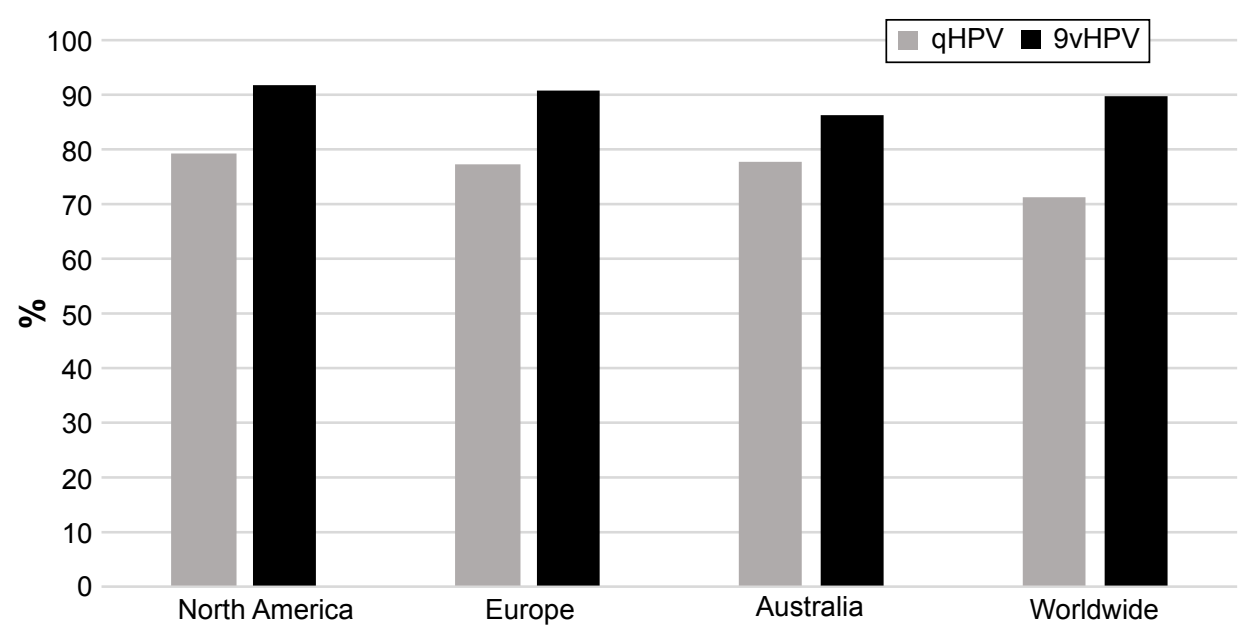

Figure 6 Estimated percentage of cervical cancers attributable to HPV types included in the qHPV and 9vHPV, in North America, Europe, Australia, and worldwide. Note: Data from Zhai and Tumban. ${ }^{24}$

Abbreviation: HPV, human papillomavirus.

2008-2012, showed that of the 38,793 cancers that occurred each year in the USA at relevant anatomic sites, approximately 30,700 could be attributed to HPV. Of these, 24,600 cancers were attributable to HPV types 16 and 18, and 28,500 were attributable to 9vHPV types. In particular, on average, the additional attributable fraction covered by $9 \mathrm{vHPV}$ accounted for $14.7 \%$ of cervical cancer, $18.3 \%$ of vaginal cancer, $14.2 \%$ of vulvar cancer, $9.0 \%$ of penile cancer, $8.2 \%$ of both anal and rectal cancers, and $5.7 \%$ of oropharyngeal cancers. ${ }^{25}$

Moreover, projected reduction of CIN 2/3 and low-grade intraepithelial lesions due to the broader coverage of 9HPV may lead to a stronger impact on screening programs. ${ }^{26}$

\section{Conclusion}

The 9vHPV was licensed by the FDA in December 2014 and by the European Commission in June 2015.

Efficacy and/or immunogenicity of 9vHPV has been assessed in eight clinical studies. The 9vHPV vaccine induced a very robust immune response against all vaccine types, with seroconversion rates close to $100 \%$. No significant interference was demonstrated when the vaccine was coadministered with other vaccines used for adolescent immunization. The use of 9vHPV in a group of women previously vaccinated with qHPV showed good immunogenicity, even though the response to the new types included in the 9vHPV vaccine was higher in women not previously vaccinated with qHPV. In conclusion, the expected efficacy of $9 \mathrm{vHPV}$ is comparable to that observed so far with qHPV, but extended to the HPV types $31,33,45,52$, and 58 .

This is the third vaccine available on the global market for prevention of persistent infection and disease caused by
$\mathrm{HPV}$, after a bivalent and a quadrivalent vaccine. Built on the clinical experience accumulated with the qHPV, the anticipated impact of 9vHPV is linked to the broader coverage against five additional types of high-risk HPV, since crossprotection toward HPV types not included in the vaccine is not warranted. Due to the strong differences between the bivalent and the nonavalent vaccine, no direct comparison is possible at present. In fact, the expected impact of the bHPV might be linked to some cross-protection toward HPV types not included in the vaccine. Such cross-protection has been demonstrated in the early postmarketing phase, but the longterm impact of cross-protection is at present debatable. ${ }^{27,28}$

Large clinical studies have demonstrated that 9vHPV and qHPV are comparable in terms of immunogenicity and efficacy against persistent infection and cervical invasive disease. Efficacy against vulvar and vaginal disease due to the new types included in the 9vHPV has also been demonstrated, even though wide confidence intervals suggest continuous evaluation to increase the number of observations and consequently the power of the study. Switching from qHPV to $9 \mathrm{vHPV}$ in the national programs may warrant a significantly higher impact on HPV-related cancers both in females and males. It is hard to precisely assess the expected impact of the additional coverage provided by the five new vaccine types in the long run, since the prevalence of HPV types may change in the population, which is also under the pressure of HPV vaccination programs. ${ }^{29}$ Moreover, no strong evidence has been collected so far on the size of the herd immunity effect produced by the different vaccination strategies and by the use of different vaccine products. The immune response to the new vaccine types included in the $9 \mathrm{vHPV}$ is optimal in naïve population. Previous vaccination with qHPV seems 
to also reduce the immune response to subsequent 9vHPV vaccination with regard to the five additional types. Since there is no certain correlate of protection with antibody levels, the clinical significance of such an observation is unclear. However, revaccination of individuals already immunized with either bHPV or qHPV - with the scope of providing protection against the additional five types - should not be considered a good policy option. ${ }^{9}$

The safety profile of 9vHPV is very good and is comparable to qHPV. Swelling and pain at the site of injection can be higher after 9vHPV vaccination, especially when coadministered with other adolescent vaccinations. Such evidence should be taken into consideration during mass campaigns, since higher local reactogenicity might reduce compliance and increase the risk of psychogenic reactions to vaccination. ${ }^{30,31}$

In conclusion, the availability of a new vaccine product represents an additional opportunity for implementing effective HPV vaccination programs. The duration of protection of HPV vaccines, potential cross-protection toward nonvaccine types, and interference between different vaccine products are still under debate in the scientific community. Continuous surveillance and ad hoc studies to better know the circulation of HPV types in the population are paramount for monitoring the effectiveness and the overall impact of the different vaccine products. The choice of the right vaccine product should be guided by local considerations including the main target of the program (cervical cancer, other HPVrelated cancers, warty disease), epidemiological situation, and pharmacoeconomic assessment.

\section{Disclosure}

The author reports no conflicts of interest in this work.

\section{References}

1. Rigoni-Stern D. Fatti statistici relative alle mallatie cancrosi che servirono de base alla poche cose dette dal dott. [Surgical facts, related to cancer diseases]. G Servire Progr Path Tera. 1842;2:507-517. Italian.

2. Zur Hausen H, Gissmann L, Steiner W, Dippold W, Dregger I. Human papilloma viruses and cancer. Bibl Haematol. 1975;43:569-571.

3. Zur Hausen H. Oncogenic herpes viruses. Biochim Biophys Acta. 1974; 417(1):25-53.

4. Muñoz N, Bosch FX, de Sanjosé S, et al. Epidemiologic classification of human papillomavirus types associated with cervical cancer. $N$ Engl J Med. 2003;348(6):518-527.

5. International Agency for Research on Cancer, World Health Organisation. IARC Monographs on the Evaluation of the Carcinogenic Risks to Human, 2016. Available from: http://monographs.iarc.fr/ENG/ Classification/. Accessed June 24, 2016.

6. World Health Organization. Immunisation Vaccines and Biologicals. Countries using HPV vaccines. Available from: http:/www.who.int/ immunization/diseases/hpv/decision_implementation/en/. Accessed June 24, 2016.
7. Stillo M, Carrillo Santisteve P, Lopalco PL. Safety of human papillomavirus vaccines: a review. Expert Opin Drug Saf. 2015;14(5):697-712.

8. GAVI. Human Papillomavirus Vaccine Support. Available from: http://www.gavi.org/support/nvs/human-papillomavirus/. Accessed June 24, 2016.

9. European Centre for Disease Prevention and Control. ECDC Vaccine Scheduler. Available from: http://vaccine-schedule.ecdc.europa.eu/ Pages/Scheduler.aspx. Accessed September 6, 2016.

10. Centers for Disease Control and Prevention. US Child and Adolescent Schedule. Available from: https://www.cdc.gov/vaccines/schedules/ hcp/imz/child-adolescent.html. Accessed September 6, 2016.

11. Public Health Agency of Canada. Update On Human Papillomavirus (HPV) Vaccines. Canada Communicable Disease Report. 2012;38:9.

12. Petrosky E, Bocchini JA, Hariri S, et al; Centers for Disease Control and Prevention. Use of 9-valent human papillomavirus (HPV) vaccine: updated HPV vaccination recommendations of the advisory committee on immunization practices. MMWR Morb Mortal Wkly Rep. 2015; 64(11):300-304.

13. European Medicines Agency. Gardasil 9. Authorisation Details. Available from: http://www.ema.europa.eu/ema/index.jsp?curl=pages/ medicines/human/medicines/003852/human_med_001863. jsp\&mid=WC0b01ac058001d124. Accessed June, 2016.

14. Joura E, Giuliano AR, Iversen O-E, et al. A 9-valent HPV vaccine against infection and intraepithelial neoplasia in women. $N$ Engl J Med. 2015;372(8):711-723.

15. Vesikari T, Brodszki N, van Damme P, et al. A randomized, double-blind, Phase III study of the immunogenicity and safety of a 9-valent human papillomavirus 11 virus-like particle vaccine (V503) versus gardasil ${ }^{\circledR}$ in 9-15-year-old girls. Pediatr Infect Dis J. 2015;34(9):992-998.

16. Van Damme P, Meijer CJ, Kieninger D, et al. A Phase III clinical study to compare the immunogenicity and safety of the 9-valent and quadrivalent HPV vaccines in men. Vaccine. 2016;34(35):4205-4212.

17. European Medicines Agency. Gardasil 9. Summary of Product Characteristics. Available from: http://www.ema.europa.eu/docs/en_GB/ document_library/EPAR_-_Product_Information/human/003852/ WC500189111.pdf. Accessed June 24, 2016.

18. Van Damme P, Olsson SE, Block S, et al. Immunogenicity and safety of a 9-valent HPV vaccine. Pediatrics. 2015;136(1):e28-e39.

19. Castellsagué X, Giuliano AR, Goldstone S, et al. Immunogenicity and safety of the 9-valent HPV vaccine in men. Vaccine. 2015;33(48): 6892-6901.

20. Garland SM, Cheung TH, McNeill S, et al. Safety and immunogenicity of a 9-valent HPV vaccine in females 12-26 years of age who previously received the quadrivalent HPV vaccine. Vaccine. 2015;33(48): 6855-6864.

21. Schilling A, Parra MM, Gutierrez M, et al. Coadministration of a 9-valent human papillomavirus vaccine with meningococcal and Tdap vaccines. Pediatrics. 2015;136(3):e563-e572.

22. Kosalaraksa P, Mehlsen J, Vesikari T, et al. An open-label, randomized study of a 9-valent human papillomavirus vaccine given concomitantly with diphtheria, tetanus, pertussis, and poliomyelitis vaccines to healthy adolescents 11-15 years of age. Pediatr Infect Dis J. 2015;34(6):627-634.

23. Markowitz LE, Dunne EF, Saraiya M, et al; Centers for Disease Control and Prevention. Human papillomavirus vaccination: recommendations of the Advisory Committee on Immunization Practices (ACIP). MMWR Recomm Rep. 2014;63(RR05):1-30.

24. Zhai L, Tumban E. Gardasil-9: a global survey of projected efficacy. Antiviral Res. 2016;130:101-109.

25. Centers for Disease Control and Prevention. Human papillomavirusassociated cancers - United States, 2008-2012. MMWR Morb Mortal Wkly Rep. 2016;65(26):661-666.

26. Fischer S, Bettstetter M, Becher A, et al. Shift in prevalence of HPV types in cervical cytology specimens in the era of HPV vaccination. Oncol Lett. 2016;12(1):601-610.

27. Riethmuller D, Jacquard AC, Lacau St Guily J, et al. Potential impact of a nonavalent HPV vaccine on the occurrence of HPV-related diseases in France. BMC Public Health. 2015;15:453. 
28. Draper E, Bissett SL, Howell-Jones R, et al. A randomized, observerblinded immunogenicity trial of Cervarix ${ }^{\circledR}$ and Gardasil ${ }^{\circledR}$ Human Papillomavirus vaccines in 12-15 year old girls. PLoS One. 2013;8(5): e61825.

29. Malagón T, Drolet M, Boily MC, et al. Cross-protective efficacy of two human papillomavirus vaccines: a systematic review and meta-analysis. Lancet Infect Dis. 2012;12(10):781-789.
30. Clements CJ. Mass psychogenic illness after vaccination. Drug Saf. 2003;26(9):599-604.

31. Clements CJ. Gardasil and mass psychogenic illness. Aust NZJ Public Health. 2007;31(4):387.

\section{Publish your work in this journal}

Drug Design, Development and Therapy is an international, peerreviewed open-access journal that spans the spectrum of drug design and development through to clinical applications. Clinical outcomes, patient safety, and programs for the development and effective, safe, and sustained use of medicines are the features of the journal, which has also been accepted for indexing on PubMed Central. The manuscript management system is completely online and includes a very quick and fair peer-review system, which is all easy to use. Visit http://www.dovepress.com/testimonials.php to read real quotes from published authors.

Submit your manuscript here: http://www.dovepress.com/drug-design-development-and-therapy-journal 